\title{
RISQUE D'INONDATION DANS LA VALLEE DE NYOS
}

\author{
Mesmin Tchindjang* et Njilah Isaac Konfor** \\ *Laboratoire de Géomorphologie, Département de Géographie, \\ Université de Yaoundé I, B.P. 30464 Yaoundé \\ **Faculté des Sciences, Université de Yaoundé I, B.P. 812 Yaoundé, Cameroun.
}

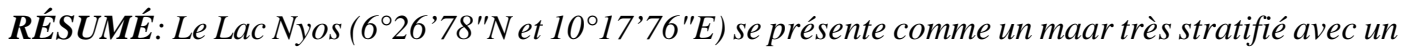
barrage intensément fracturé. Il occupe une topographie d'alvéoles granitiques doublées d'intrusions rhyolitiques et trachytiques. Ce lac situé à 1100m d'altitude et couvrant une superficie de 275 ha s'encaisse dans des granites très fracturés et cataclasés dont les falaises surplombent de $100 \mathrm{~m}$ le niveau de l'eau et le plancher lacustre. Il a une forme rectangulaire avec un exutoire NO barré par des pyroclastites à grano classement latéral et qui se sont consolidés. Ce barrage naturel d'éjectas grossiers protège les populations et les installations en aval, sa rupture ferait au moins 10000 morts du Cameroun au Nigeria selon McKIE (2000). En effet avec ses $2 \mathrm{~km}$ de long, $1 \mathrm{~km}$ de large et $210 \mathrm{~m}$ de profondeur, le lac Nyos contient 3 milliards de $\mathrm{m}^{3}$ d'eau et il retient beaucoup de gaz dissous notamment le dioxyde de carbone.
\end{abstract}

Mots-clés: barrage de pyroclastites, inondation, lac Nyos, risques

\begin{abstract}
The maar Nyos, situated at latitude 6 ${ }^{\circ} 26^{\prime} 78^{\prime \prime} \mathrm{N}$ and $10^{\circ} 17^{\prime} 76^{\prime \prime} E$ along the Cameroon Volcanic Line is well known for its stratification from bottom to top. It lies on a granitic topography at $1100 \mathrm{~m}$ above sea level. The granitic cliff exposed to the west of the lake rises $100 \mathrm{~m}$ above the water level. The lake waters are held behind to the north by a natural pyroclastic dam on which water overflows creating semi giant potholes. The overflow forms a waterfall of about 30-40m. This dam is highly and densely fractured (13 fractures of $23-40 \mathrm{~m}$ long). The breakdown of this dam can create floods that will drawn over 10.000 people in both Cameroon and Nigeria (according to McKIE, 2000). The lake contains 3 billions $m^{3}$ of water. At the bottom, the concentration of carbon dioxide is $85 \mathrm{~m} / 10 \mathrm{~m}^{3}$ of water. The dam remains a great potential danger to the population, aggravated by the high concentration of carbon dioxide in the lake emanating from the mantle which can lead to another gas disaster. The tectonic instability or ever a slight earth tremors can break the dam and cause floods downstream. A volcanic eruption might even worsen the situation. This paper presents the results of a recent research carried out on the lake after the onset of the first phase of the degassing project.
\end{abstract}

Key-words: floods, lake Nyos, pyroclastic dam, risks.

\section{INTRODUCTION}

Le lac Nyos, situé dans la Province du Nord-Ouest (fig.1a), occupe le flanc Nord du mont Oku (fig.1b) dont le volcanisme s'étale de 31MA jusqu'à l'actuel (Njilah, 1991). Les laves anciennes issues du massif d'Oku vont des basaltes alcalins (hawaïtes) aux trachytes et rhyolites. Le volcanisme récent de ce massif a édifié des cratères et cônes de scories partiellement détruits et dont certains abritent des lacs en l'occurrence les lacs Nyos, Oku, Njupi etc.(fig.1b). Le lac Nyos qui est l'objet de la présente étude date de $400 \pm 100$ B.P. (Lockwood et Rubin, 1989).
Depuis la lointaine éruption de 1986 au cours de laquelle les scientifiques sont restés indécis et partagés sur l'origine du gaz carbonique dissous dans l'eau, l'unanimité tend à se faire aujourd'hui et l'on reconnaît le caractère hydrodynamique du lac Nyos, ainsi que l'instabilité géologique de la région qui ne rend pas facile une modélisation des risques. Dès lors, le maar Nyos, quoique isolé dans cette région devenue lugubre et abandonnée, tend à devenir le nouveau pôle de la recherche scientifique dans le domaine des catastrophes géoenvironnementales au Cameroun. 


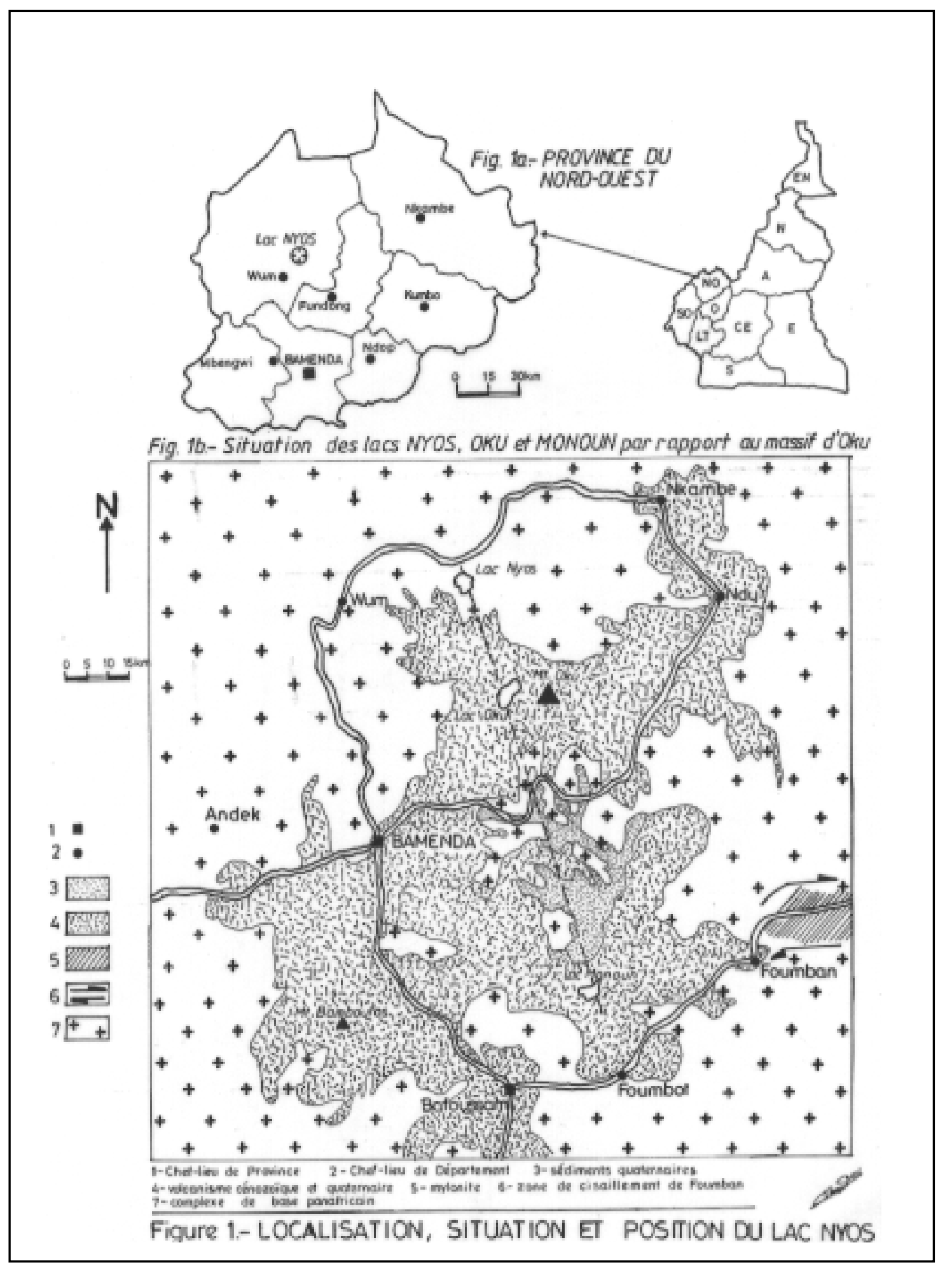




\section{La catastrophe du lac Nyos: rappel.}

L'éruption du lac Monoun le 16 août 1984 qui tua 37 personnes et quelques animaux (Sigurdson et al., 1987) fut suivi deux ans plus tard par celle du lac Nyos. En effet, le jeudi 21 août 1986, une grande explosion dans le lac libéra un important volume de gaz plus lourd que l'air. Ce gaz emprunta la trajectoire des vallées environnants pour atteindre les villages avoisinants, tuant plus de 1700 personnes ainsi que beaucoup d'animaux domestiques et sauvages. Ce fut la plus grande catastrophe gazeuse jamais enregistrée dans le monde entier (Kusakabe, 1989). Le caractère singulier de cette catastrophe attira l'attention de la Communauté internationale sur ce petit lac jadis inconnu du Nord-Ouest du Cameroun (fig.1a; Lockwood et Meyer, 1989). La catastrophe du lac Nyos demeure à ce l'événement naturel le plue tragique qui ait frappé le Cameroun à cause de nombreux décès, de pertes des animaux sauvages et de déplacements des populations. Trois villages situés à basse altitude furent particulièrement atteints: Nyos, environ 1200 décès; Subum, environ 300 décès et Cha, environ 200 décès (fig. 3). Les villages éloignés du lac Nyos et situés au nord de Cha, dans le bassin de Mbum, enregistrèrent ensemble 21 décès (village Fang, Mashi, Mundambili et Mufo). Au lendemain de la catastrophe, l'on dénombra au total 874 rescapés intoxiqués et qui ont été conduits dans les hôpitaux alentours. Ceuxci souffraient des lésions corporelles, des brûlures épidermiques ponctuelles, des irritations des muqueuses respiratoires, des affections pulmonaires et de paralysies (monoplégie et paraplégie; MORIN et PAHAI, 1987). Au total, 4434 personnes furent déplacées de leurs villages et maisons.

\section{II-OBSERVATIONSET RESULTATS}

\section{A) La plate forme de dégazage}

Le Lac Nyos (fig. 1a-1b) constitue avec le Lac Monoun (à l'état des connaissances actuelles) les seuls maars dangereux à gaz carbonique dissous, connus à ce jour au Cameroun. Depuis 1990, une équipe scientifique a entrepris une expérience à partir de tuyaux plastiques de polyéthylène pour comprendre le mécanisme de fonctionnement et d'expansion du $\mathrm{CO}_{2}$ dissous dans l'eau. L'expérience s'est avérée concluante et dès 1995,. des colonnes ont été conçues et réalisées pour dégazer le Lac Nyos. Leur mécanisme est simple. On monte une plate forme (fig. 2a) qui flotte sur le lac et au milieu de laquelle on fait déboucher des tuyaux enfoncés sous l'eau $(210 \mathrm{~m}$ de profondeur). Sur ces installations simples, 3 vannes ont été placées:
- $\quad$ une vanne latérale à 100 m qui peut faire projeter l'eau à $10 \mathrm{~m}$ de haut,

- $\quad$ une vanne centrale à $140 \mathrm{~m}$, qui peut propulser l'eau à plus de $30 \mathrm{~m}$; le bout de la colonne est à $203 \mathrm{~m}$.

- $\quad$ une vanne en surface, $2 \mathrm{~m}$ de propulsion

A partir de ces vannes, l'on a constaté que le $\mathrm{CO}_{2}$ dissous (depuis 500 à 5000 B. P.) varie des profondeurs vers la surface en même temps que la concentration du $\mathrm{CO}_{2}$ et la pression sont importantes entre 190 et $210 \mathrm{~m}$. Le taux de recharge obtenu depuis 1990 est de $0,66 \%$ par an et la pression est passée de 10 bars en 1990, 12 en 1992 et 14 en 1999 11. Il a été constaté qu'en 11 ans, l'eau serait complètement saturée en dioxyde de carbone, au rythme de 5millions de $\mathrm{m}^{3}$ par an. D'où l'apport des colonnes installées.

Les vannes installées permettent de contrôler le dégazage du lac à travers des colonnes de $70 \mathrm{~m}$ de long, 20 de large, $16 \mathrm{~cm}$ de diamètre. Le jet d'eau qui monte et parvient en surface atteint $48 \mathrm{~m}$ de hauteur (fig.2a) de propulsion à partir de la plate forme; et, cette eau est sursaturée et constituée de $80-90 \%$ de $\mathrm{CO}_{2}$. En effet l'équipe de l'IRGM en place, qui travaille en collaboration avec les chercheurs japonais, britanniques et américains, nous a affirmés que le lac Nyos montre une teneur importante de $85 \mathrm{~m} / 10 \mathrm{~m}^{3}$ de $\mathrm{CO}_{2}$ en dehors du méthane et de l'hydrogène sulfuré. ( Par ailleurs, Tazieff et al. (1986) que cite Morin (1986) signale que la concentration peut être de 131 à $220 \mathrm{~m}$ de profondeur, 91 à $150 \mathrm{~m}$ et $7,21 \mathrm{de} \mathrm{CO}_{2}$ à $120 \mathrm{~m}$ ). Le système d'autopompe par lequel le dégazage se réalise est entretenue par l'énergie solaire à travers des plaques solaires qui rechargent des batteries d'alimentation.

L'équipe pluridisciplinaire qui s'occupe de ce dégazage est en train d'installer un observatoire complet avec relais satellitale. Pour ce faire, il est prévu 5 plates-formes flottantes pour réaliser le dégazage complet du lac au bout de 5 ans. Par ailleurs, l'IRGM a installé un détecteur sonore de gaz carbonique; en cas de concentration anormale du dioxyde de carbone, l'appareil émet un signal lumineux rouge et l'alarme retentit pour obliger les populations à fuir et se réfugier sur les points hauts du relief (le même système a été installé au lac Monoun qui attend également son observatoire).

\section{B) Le barrage de pyroclastites et sa fracturation: danger de rupture}

\section{1) - Synthèse de la fracturation dans la région de Nyos}

Les fractures relevées sur la dalle font partie d'un vaste ensemble tectonique couvrant toute la région de Nyos et 


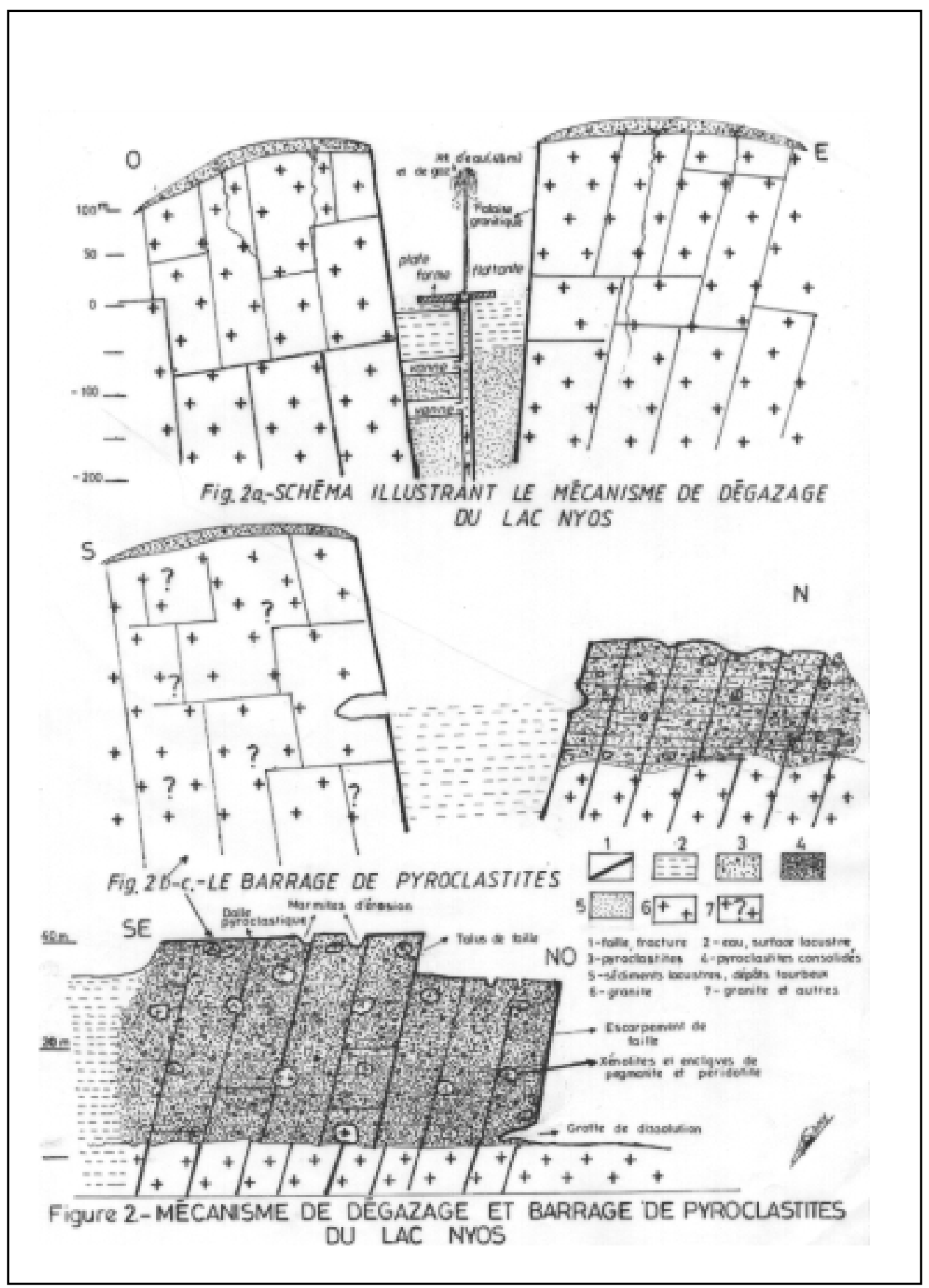


organisé en 2 couloirs principaux décrits par MORIN et PAHAI. (1986). Il s'agit du couloir de Kam-Subum orienté OSO-ENE sur une faille $\mathrm{N} 70^{\circ} \mathrm{E}$ assimilée à une virgation des vieux linéaments panafricains $\mathrm{N} 20^{\circ} \mathrm{E}$ et qui compte avec les accidents $\mathrm{N} 65-80^{\circ} \mathrm{E}$ pour $30 \%$ de directions (MORIN et al. (1986). Dans ce couloir de 0,5-1km de large, les altitudes vont de 820 à $900 \mathrm{~m}$. Vers le NO, les failles retrouvent à travers la rivière Mbum la direction panafricaine. La rivière Mbum à l'Ouest du lac Nyos et la rivière Kimbi à l'Est, s'inscrivent sur des lignes de faille $\mathrm{N} 20-30^{\circ} \mathrm{E}$ au Sud et $\mathrm{N} 02-07^{\circ}, 170-175^{\circ} \mathrm{E}$ (failles subverticales) au Nord. Elles constituent ainsi un faisceau de failles dont le centre où s'inscrit le lac Nyos est caractérisé par un abaissement des altitudes $(1300 \mathrm{~m}$ au
Sud à-600m au Nord), alors que, les bordures occidentales et orientales sont relevées (1300-1900m). Ce faisceau prolonge la structure camerounaise jusqu'au Nigeria. Le second couloir est celui de la Jonga affluent de la Kimbi, tributaire de la Katsina. Ce couloir de direction $\mathrm{N} 25^{\circ} \mathrm{E}$ et caractérisant des accidents variant de N25 à N35 $\mathrm{E}$ (fig. 3), s'abaisse de 962m (Bwabwa-Sowe) au SO à 798-788m (Djingbe Subum) au NE. Franchement orienté SO - NE, il est assez large $(1-2 \mathrm{~km})$ et vers le Nord à hauteur de Nkannye, la faille principale prend une virgation $\mathrm{N} 112^{\circ} \mathrm{E}$. La Kimbi conflue vers la Katsina à une altitude de $300 \mathrm{~m}$. Temdjimet Tchoua (1991), sur la base d'une étude de photographies aériennes signalent 3 systèmes de failles:

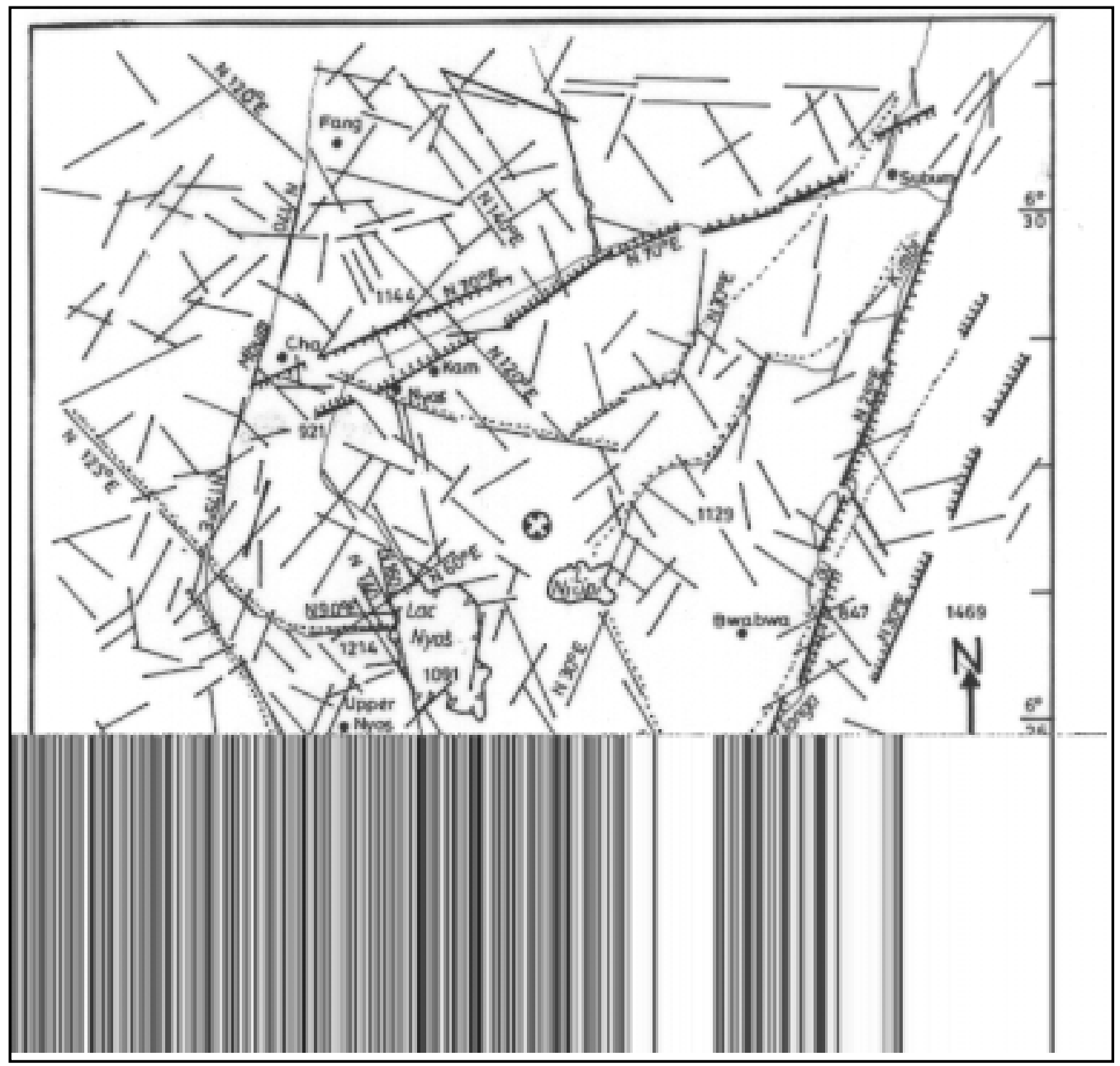

Fig 3: Ce barrage qui bloque l'écoulement des eaux vers l'exutoire de vidange du lac est très stratifié. On observe les couches déposées lit par lit comme dans un bassin sédimentaire. On observe aussi en dessous l'attaque chimique de ce barrage par les eaux du lac bien chargées en $\mathrm{CO} 2$ qui dissolvent les produits pyroclastiques. Nous avons compté plus d'une cinquantaine de strates. En surface, ce barrage se moule en dalle et en contrebas une cavité souterraine liée à la dissolution chimique voire karstique ou pseudo karstique existe. Ce barrage constitue un risque potentiel en cas de rupture par un séisme, une éruption volcanique etc. 
$>$ Un système commandé par la $\mathrm{N} 30^{\circ} \mathrm{E}$ qui est la résultante des directions des accidents variant du $\mathrm{N} 25^{\circ}$ au $\mathrm{N} 35^{\circ} \mathrm{E}$ (fig. 3). Ce premier système se termine par un couloir qui sépare le secteur de Wum (Nyos) à l'Ouest de celui de Nkambe à l'Est (fig. 1b et 3).

$>$ La direction $\mathrm{N} 70^{\circ} \mathrm{E}$ qui se moule en un couloir de fracturation (plaine de Kam-Nyos selon Temdjim et Tchoua) orienté OSO-ENE. Cette tranchée tectonique sépare le district volcanique de Nyos des massifs granitiques septentrionaux (fig. 1b et 3 ).

$>$ Le troisième système représenté par la $\mathrm{N} 120^{\circ} \mathrm{E}$ est la moyenne des directions allant de $\mathrm{N} 115^{\circ}$ à $\mathrm{N} 135^{\circ} \mathrm{E}$ auxquelles il faut ajouter les directions $\mathrm{N} 140^{\circ} \mathrm{E}, \mathrm{N} 160^{\circ} \mathrm{E}$ qui délimitent le lac Nyos à l'Ouest (fig. 3).

Le lac Nyos est donc encadré par 3 systèmes de failles dont 2 couloirs de fracturation. Les deux couloirs sont parcourus par une série de failles qui les décalent en échelons, notamment: N115, $152,130,150,160$ et $175^{\circ} \mathrm{E}$. Le couloir de Kam-Subum rejoint celui de la Jonga à $6^{\circ} 28^{\prime} 40^{\prime \prime} \mathrm{N}$ et $10^{\circ} 20^{\prime} 50^{\prime \prime}$ E où ils y forment une zone déprimée de $3 \times 2 \mathrm{~km}$. En cas de rupture du barrage de pyroclastites, les eaux descendraient par le couloir Kam-Subum pour rejoindre la Kimbi au NE ou secondairement pour rejoindre la Mbum à l'Ouest par Cha; ces deux principaux collecteurs de la région sont des affluents de la Katsina au Nigeria.

\section{2) - Structure et fracturation du barrage et du lac}

Pour ce qui est du barrage de pyroclastites, nos observations sur le terrain (3 avril 2001) nous révèlent plus d'une vingtaine de couches de 5 à $15 \mathrm{~cm}$ d'épaisseur de pyroclastites stratifiés obliquement et plongeant vers le Nord. Ces strates, qui traduisent des séquences pulsatives, apparaissent intensément fracturées et traversées de l'Est à l'Ouest par 13 fractures bien visibles de $40 \mathrm{~m}$ de long et de direction $\mathrm{N} 80-90^{\circ} \mathrm{E}$. Au niveau de la $12^{\text {ème }}$ fracture, on observe un talus de faille de 1,5 m de dénivelée alors que la $13^{\text {ème }}$ fracture matérialise un escarpement de faille de 30 à $40 \mathrm{~m}$ de dénivelées taillé dans les pyroclastites mélanocrates (fig.2b-c) et surplombant un couloir tectonique modelé dans les granites; et où débouche une rivière souterraine qui par dissolution chimique des pyroclastites a percé le barrage comme dans un karst. Les fractures que nous avons dénombrées en dehors des 2 dernières présentées plus haut ont une longueur visible de $23 \mathrm{~m}$, une profondeur de 15 à $22 \mathrm{~cm}$ et un écartement de lèvres de 7 à $15 \mathrm{~cm}$. La densité est de 1 fracture tous les $3 \mathrm{~m}$ en dehors de fissures.

\section{3) - Risques sismiques de rupture du barrage}

La fracturation intense que nous venons d'évoquer accroît les risques de séisme. En effet, un tel système rhéologique de fractures entrecroisées avec décalage en échelons fait de Nyos un champ de failles et de contraintes dont les accidents principaux $\mathrm{N} 20^{\circ}, \mathrm{N} 70^{\circ}$ et $\mathrm{N} 90^{\circ} \mathrm{E}$ avec leurs transverses $\mathrm{N} 160-170^{\circ} \mathrm{E}$ sont accompagnés de décrochements $\mathrm{N} 120-140^{\circ} \mathrm{E}$. La succession de contraintes tectoniques distensives se poursuit de nos jours par la libération du $\mathrm{CO}_{2}$ mantellique qui remonte par les fractures ouvertes. Les fractures du barrage qui ne datent que de 400 à 500BP et qui n'ont jamais été signalées par aucune étude constituent une preuve éloquente de la permanence tectonique. La continuité du faisceau Mbum-Kimbi (N20 E ) qui a $10-20 \mathrm{~km}$ de large auquel se joint le faisceau KamSubum et Acha-Bwabwa $\left(\mathrm{N} 70^{\circ} \mathrm{E}\right)$ orienté ONO-ESE qui ferme le premier faisceau au Sud se rompt de failles transverses $160-170^{\circ} \mathrm{E}$ qui fonctionne comme des décrochements horizontaux ainsi que des failles listriques apparemment plus récentes. Celles-ci sont mises en évidence par la faille qui accidente la rivière Tsobu de direction $\mathrm{N} 90^{\circ} \mathrm{E}$ qui vidange en partie le lac vers l'Ouest. Cette direction de faille de la Tsobu rejoint celle que nous avons relevée sur la dalle du barrage (photos 1 et 2) de pyroclastites. Cette fracturation est une mise évidence des possibilités de rupture du barrage. De plus, elle est importante parce que les conduits souterrains de dissolution et d'écoulement karstiques exploitent ou suivent toujours les points de fragilisation de la roche: points de stratification, diaclases, fissures, failles et le réseau tectonique de Nyos est en général orthogonal.

\section{4) - Dissolution des pyroclastites par $\mathrm{le}^{\mathrm{CO}_{2}}$ et rupture du barrage.}

Le barrage (photo 3$)$ de pyroclastites $\left(6^{\circ} 26^{\prime} 72^{\prime \prime} \mathrm{N}\right.$ et $10^{\circ} 17^{\prime} 66^{\prime \prime} \mathrm{E}$ ) se moule sur une dalle fracturée (photo 1 ) et trouée de marmites d'érosion (photo 2) liées au débordement des eaux du lac qui remontent à la faveur des pluies diluviennes de saison pluvieuse $(1800 \mathrm{~mm} / \mathrm{an})$ et de la forte concentration de $\mathrm{CO}_{2}$ (et d'autres gaz) qui a un pouvoir de dissolution chimique élevé, $(\mathrm{pH}=5$ en surface et $>6$ en profondeur). On sait que les réseaux karstiques ou pseudo karstiques sont marqués en profondeur de cavernes et parcourus de rivières souterraines; les cupules, vasques et marmites relevées (photo 2) sont des formes de moyenne échelle du modelé des lapiés, des dolines et des poljés calcaires des milieux tempérés et tropicaux. La mise en place d'un modelé karstique obéit à plusieurs conditions dont deux apparaissent capitales: la composition chimique de la roche et l'agressivité de l'eau chargée de $\mathrm{CO}_{2}$. 


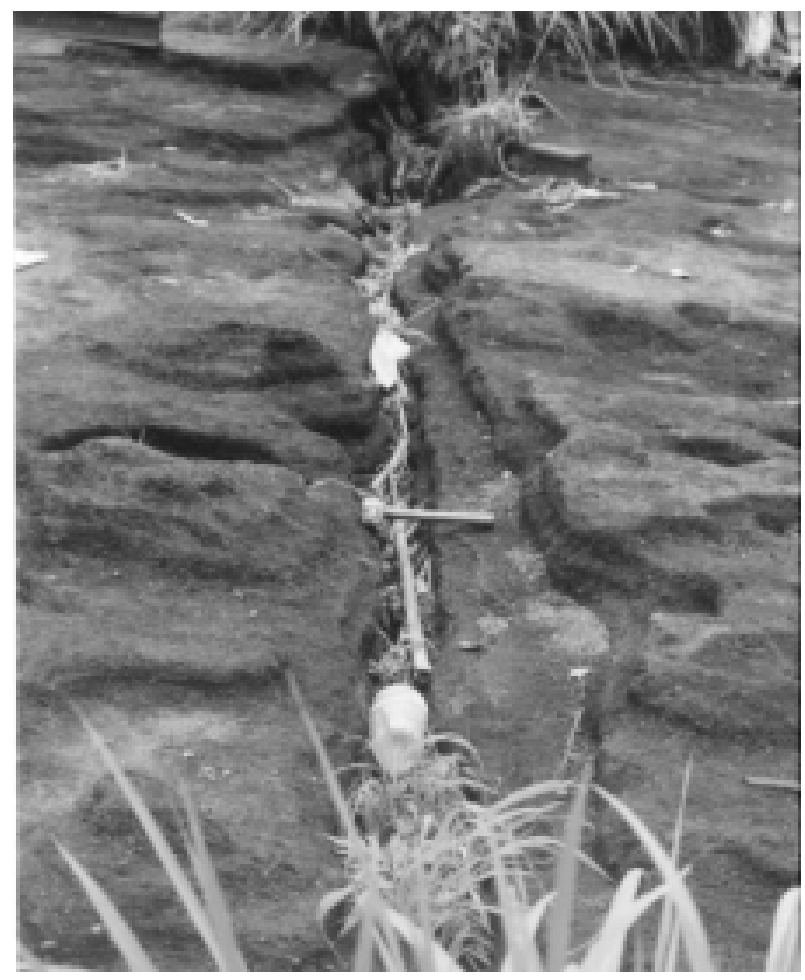

Photo 1: On observe la dalle pyroclastique consécutive au barrage consolidée. Elle est traversée par des fractures dont l'écartement est visible ici (flèche) et qui sont remplies de débris de toutes sortes. Photo Tchindjang, 3 avril 2001

\section{a)-La composition chimique de la roche}

Les pyroclastites qui barrent les eaux de la façade septentrionale du lac Nyos sont constitués de scories, de débris de basalte, de lapillis grossiers, des xénolites de granite, des enclaves de pegmatite et de péridotite enrobés dans une gangue basaltique, le tout consolidé. Tazieff et al. (1986) signale des carbonatites dans la région de Nyos. Cette nature pétrographique contient en elle-même des germes d'explication de la composition chimique des pyroclastites. Ceux-ci sont mélanocrates à holomélanocrates contenant beaucoup de ferromagnésiens qui confèrent une valeur élevée au calcium $(\mathrm{CaO})$ et au Magnésium ( $\mathrm{MgO})$. La plupart des pyroclastites de la région du Noun autour des lacs Monoun et Petponoun ont une composition minimale moyenne de 9,50\% de $\mathrm{CaO}$ et de $8 \%$ de $\mathrm{MgO}$ pour $44 \%$ de silice (d'après les données de Wandji, 1985). Or, ces 2 éléments sont très sensibles à la présence de l'eau chargée de $\mathrm{CO}_{2}$ et c'est pour cette raison que les pyroclastites s'altèrent très vite. Cette rapide altération va se doubler ici à Nyos d'une dissolution chimique active liée à la présence des péridotites et des carbonatites. En effet, la péridotite selon Foucault et al. (1995) est une roche grenue jaune sombre huileux, ou plus souvent vert noirâtre (c'est le faciès récolté à Nyos); holomélanocrate avec $90-100 \%$ de ferromagnésiens. Elle est donc ultrabasique ou ultramafique à composition d'olivine dominante accompagnée de pyroxène, spinelle et parfois d'amphibole brune. Le faciès de Nyos est une Iherzolite à composition dominante de clino et d'orthopyroxènes. La plupart des échantillons récoltés sont altérés. Leur gisement se trouve dans le manteau supérieur. Dans le cas de Nyos, ces xénolites mantelliques affleurent en enclaves dans les basaltes. La carbonatite est également une roche magmatique grenue ayant l'aspect de calcaire cristallin et constitué à $90 \%$ de grands cristaux de carbonate soit de la calcite, soit de la calcite et de la dolomite. Elle a un gisement intrusif ou filonien ou alors elle se présente sous forme de coulées associées à des roches ultrabasiques (péridotites, kimberlites). Dans le cas des Nyos, la carbonatite est associée à la péridotite et elle se forme à partir d'un magma enrichie en $\mathrm{CO}_{2}$ et $\mathrm{Ca}$ (calcium) selon des modalités mal connues; Foucault et al. (1995). On constate donc que la composition des pyroclastites de Nyos constitue déjà un facteur propice à l'érosion chimique par dissolution à cause de la présence du $\mathrm{CO}_{2}$, du calcium, du magnésium et d'autres minéraux hydroxylés dans ces roches (amphibole etc.)

\section{b) - L'agressivité de l'eau}

L'érosion chimique (dissolution) domine les processus physiques dans les zones tropicales humides (Petit, 1990). Cette dissolution s'exprime par la formule classique $\mathrm{CaCO}_{3}$ $+\mathrm{CO}_{2}+\mathrm{H}_{2} \mathrm{O} \rightleftharpoons 2\left(\mathrm{CO}_{3} \mathrm{H}\right) \mathrm{Ca}$. Plusieurs études montrent que l'eau pure dissous peu de carbonate. Ainsi, Derruar (1974) et Petit (1990) montrent que l'eau chargée de $\mathrm{CO}_{2}$ est très agressive et mène à la formation de l'acide carbonique selon la réaction de dissolution: $\mathrm{CO}_{2}+\mathrm{H}_{2} \mathrm{O}=$ $\mathrm{H}_{2} \mathrm{CO}_{3}$ (acide carbonique). Cet acide s'associe à la présence du calcium et donne la réaction: $\mathrm{H}_{2} \mathrm{CO}_{3}+\mathrm{CaCO}_{3}=$ $\mathrm{Ca}\left(\mathrm{CO}_{3} \mathrm{H}\right) 2$. Les deux réactions s'additionnent $\left[\mathrm{CaCO}_{3}+\right.$ $\mathrm{CO}_{2}+\mathrm{H}_{2} \mathrm{O}=\mathrm{Ca}\left(\mathrm{CO}_{3} \mathrm{H}\right) 2$ ] pour donner le bicarbonate de calcium qui est très instable et particulièrement soluble dans l'eau. Tricart (1977) signale que la solubilité du bicarbonate est plus forte à basse température et que la teneur des eaux en $\mathrm{CO}_{2}$ résulte de la destruction de la matière organique qui est très abondante sous climat chaud et humide. En même temps que la décomposition chimique y est plus rapide et provoque une forte libération du $\mathrm{CO}_{2}$ dont l'excès conduit à la formation du bicarbonate de calcium (très corrosif) selon les réactions ci-dessus. 


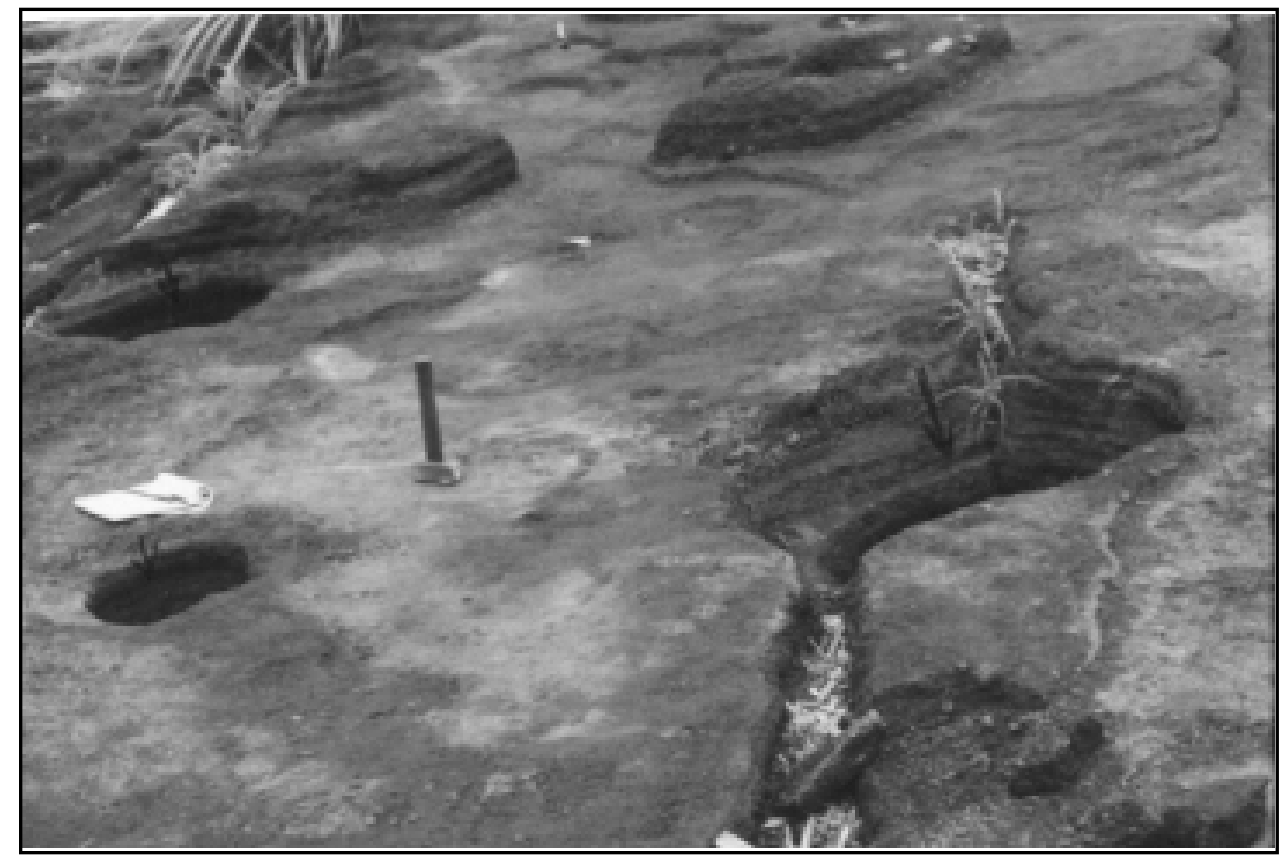

Photo 2 : Les marmites d'érosion sur pyroclastites consolidées de la dalle de pyroclastites. Ces marmites (flèche) sont le résultat d'une double attaque chimique des eaux du lac très chargées en dioxyde des carbone et des eaux descendus des versants orientaux et occidentaux qui surplombent le barrage.

Photo Tchindjang, 3 avril 2001

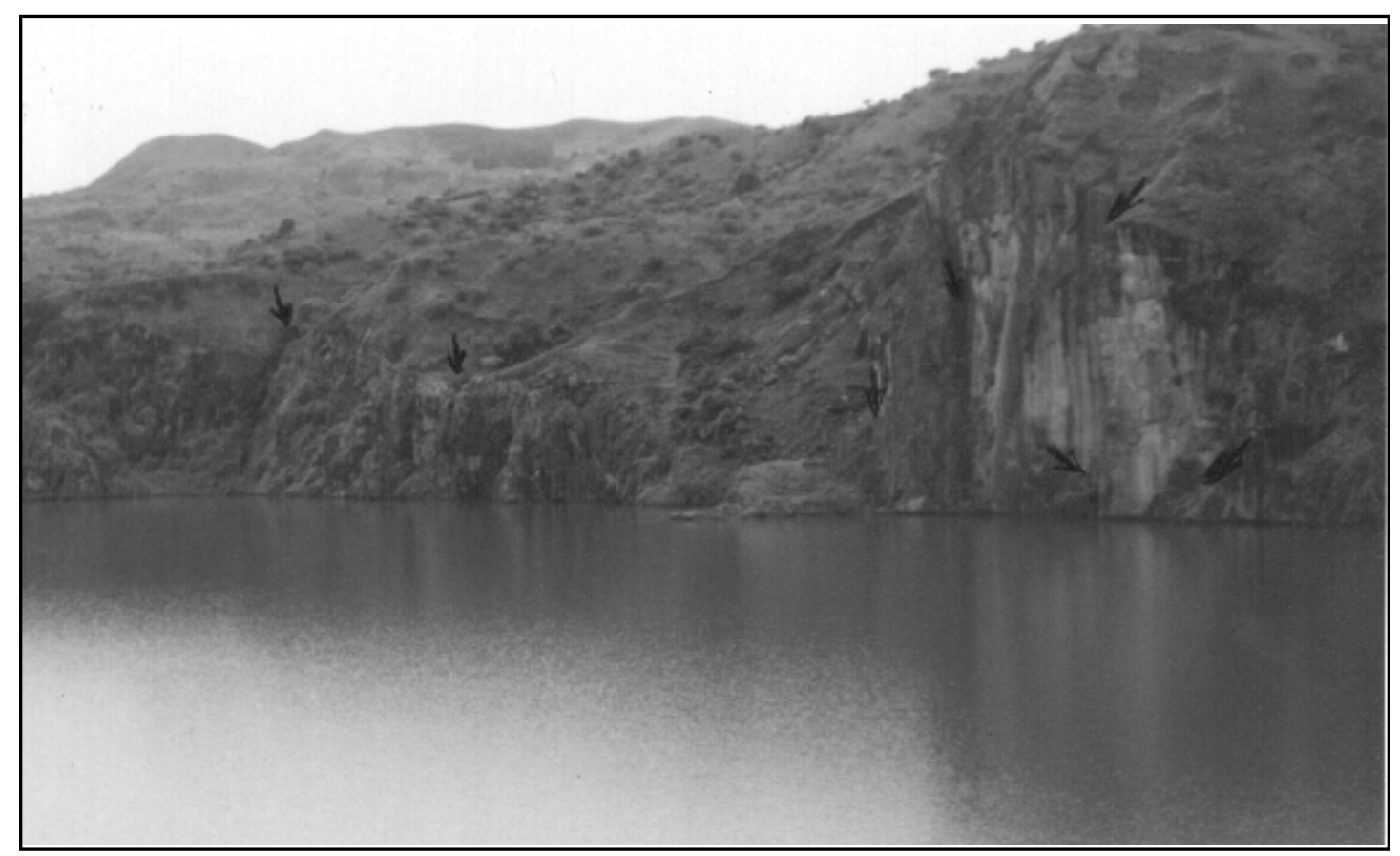

Photo 3 : la falaise granitique ci-dessus parcourue de fractures (flèches).

Photo Tchindjang, 3 avril 2001 
Derruau (1974) renchérit en montrant que le carbonate de chaux $\left(\mathrm{CaCO}_{3}\right)$ est faiblement soluble dans l'eau chimique pure et fortement dans l'eau chargée d'acide carbonique en plus d'autres (acide lactique et fulvique). Et c'est bien le cas ici au lac Nyos dont les eaux recèlent une importante quantité de $\mathrm{CO}_{2}$ dissous dont l'origine doit être élucidée.

\section{c)-les sources du $\mathrm{CO}_{2}$ et les mécanismes de dissolution à Nyos.}

Avec ces auteurs, la concentration de ce gaz dans l'eau dépend de la pression partielle du $\mathrm{CO}_{2}$ au contact de l'eau. L'eau de pluie contient peu de $\mathrm{CO}_{2}(0,8-2,5 \mathrm{mg} / \mathrm{l}$ pour une pression de $2 / 100^{\text {ème }}$ d'atmosphère); par contre, l'eau du sol en est plus riche (50-200mg/l pour une pression de 8/ $\left.100^{\mathrm{ème}}\right)$. Par conséquent, l'eau de circulation souterraine des grottes et cavernes contient 2 à $8 \%$ de $\mathrm{CO}_{2}$ et constitue la première source de dissolution Les eaux de la source thermominérale de Nyos contiennent $6,5 \mathrm{mg} / \mathrm{l}$ de calcium et $12 \mathrm{mg} / \mathrm{l}$ de magnésium pour $1000 \mathrm{mg} / 1 \mathrm{de} \mathrm{CO}_{2}$ et $19 \mathrm{mg} / \mathrm{l} \mathrm{de}$ silice seulement, (Le Marchal, 1976). En effet, Petit (1990) en signalant que la pression partielle dans le sol entre 20 et $150 \mathrm{~cm}$ atteint 45/10000e d'atmosphère en Nouvelle Guinée précise très bien que celle-ci bouleverse les données thermiques. Il ajoute qu'avec une pression de $8 / 100^{\mathrm{e}}$ d'atmosphère, la dissolution du $\mathrm{CO}_{2}$ passerait de $2,69 \mathrm{mg} /$ 1 à $0^{\circ}$ contre $1,38 \mathrm{mg} / \mathrm{l}$ à $20^{\circ}$. Il en conclut que la zone tropicale possède un potentiel "agent d'érosion" d'une capacité agressive 5 à 10 fois supérieure à celui de la zone tempérée selon les données ci-dessous:

\begin{tabular}{llll} 
& $\frac{\text { Zone froide }}{25-30 \mathrm{mg} / \mathrm{l}}$ & & Zone tropicale \\
\cline { 2 - 3 } Dalle nue & $60-80 \mathrm{mg} / \mathrm{m} / \mathrm{l}$ & $150 \mathrm{mg} / \mathrm{l}$ \\
Fissures à humus & 60 ) & $150-200 \mathrm{mg} / \mathrm{l}$ & $300-400 \mathrm{mg} / \mathrm{l}$
\end{tabular}

En plus des eaux de circulation souterraine ou de celles qui débordent du lac en saison pluvieuse, la forme en gouttière du maar Nyos prédispose la barrière de pyroclastites située à l'exutoire de collecter les eaux descendues des versants occidentales et orientales avec tous leurs produits 3 avant de les acheminer en aval par une chute de plus de $40 \mathrm{~m}$. De plus, à cause de la forte charge en $\mathrm{CO}_{2}$ dissous des eaux du lac, la présence d'une grotte de dissolution et d'écoulement karstique sous le barrage du côté de l'exutoire, accroît le danger d'une rupture en cas de séisme, car, il s'agit bien d'un phénomène pseudokarstique. C'est l'une des sources de $\mathrm{CO}_{2}$; car, avec l'altitude, les conditions de dissolution en milieu tropical sont favorables entre 1000 et $3000 \mathrm{~m}$ où on relève une abondance des ressources en eau, des ressources végétales, une forte luminosité et des températures constantes et régulières qui sont favorables aux réactions chimiques et biologiques précédemment évoquées (formation de l'acide carbonique et du bicarbonate de calcium).

Il faut ajouter à cela l'interférence des poussées tectoniques et des paléoclimats qui font évoluer cette dissolution en milieu tropical humide (comme Nyos) de $40-80 \mathrm{~mm}$ pour 1000ans selon Williams (1972) et Corbel (1970). Rossi (1980) que cite Petit (1990) mesure une dissolution de 200-300mg/ len saison humide contre 150 en saison sèche et calcule le taux moyen de dissolution à $135 \mathrm{~mm}$ par millénaire dans les reliefs du NO malgache avec $2000 \mathrm{~mm}$ de précipitations. Que l'on ne se surprenne pas de cette hypothèse, car, sur le versant opposé du barrage de pyroclastites, les populations exploitent le sel gemme (dont la consistance calcaire est reconnue) et l'on sait que la karstification tropicale dépend essentiellement de l'agressivité de l'eau et de la composition chimique des roches.

L'eau a donc un effet plus important, s'il est chargé d'acide carbonique dans des régions où les précipitations sont supérieures à $1500 \mathrm{~mm}$, comme c'est le cas ici à Nyos (1800mm). Cependant, la température de l'eau ici est relative ; car le lac étant très stratifié en profondeur ne peut y accueillir le gaz carbonique atmosphérique et par conséquent la chaleur issue du rayonnement solaire. Or, l'eau froide absorbe plus de $\mathrm{CO}_{2}$ que l'eau chaude selon les travaux de Williams (1972) et Corbel $(1962,1970)$. Derruau, (1974) renchérit en précisant le taux de dissolution suivant la température ; pour cet auteur, 11 d'eau dissous $1,01 \mathrm{mg}$ de $\mathrm{CO} 2$ à $0^{\circ}, 0,70 \mathrm{mg}$ à $10^{\circ}, 0,52 \mathrm{mg}$ à $20^{\circ}$ et $0,39 \mathrm{mg}$ à $30^{\circ}$. Petit ajoute que dissolution et saturation du carbonate passent de $475 \mathrm{mg} / 1$ à $0^{\circ}$ à $300 \mathrm{mg} / 1$ à $30^{\circ}$. Par ailleurs, au fur et à mesure que l'eau chargée d' acide carbonique prend le calcium en solution, le $\mathrm{pH}$ augmente et la solution devient plus basique et moins acide. On a là une explication au $\mathrm{pH}$ très basique des eaux profondes du lac Nyos $\mathrm{pH}>7$ en profondeur contre 5 en surface. Cependant, c'est l'acide carbonique et les autres acides (nitrique) qui réalisent dans le cas de Nyos l'attaque du calcium et du magnésium dont les eaux du lac en sont enrichies en profondeur (52\% de calcium à 150m de profondeur d'après Tazieff et al. (1986). Les explications de ces auteurs permettent de comprendre la recharge du $\mathrm{CO}_{2}$ mantellique à Nyos et la dissolution en profondeur de la dalle. Il faut ajouter à cela que la présence de la silice dans ces pyroclastites, accentue l'acidité et le pouvoir dissolvant de l'eau. Les régions tropicales de montagne comme Nyos répondent très bien à ces exigences de dissolution karstique pour un matériel pyroclastique très apte où l'agressivité de l'eau, son acidité et la rapidité de dissolution du $\mathrm{CO}_{2}$ compensent la forte température. 
De plus les montagnes sont plus avantagées dans ce domaine que les régions basses, parce que la diminution de la pression atmosphérique en montagne entraîne la diminution conséquente de la teneur en $\mathrm{CO}_{2}$ atmosphérique

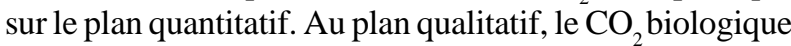
naît des racines et il est plus efficace que le $\mathrm{CO}_{2}$ atmosphérique. $\mathrm{Ce} \mathrm{CO}_{2}$ biologique existe bel et bien dans les eaux de Nyos puisque les études de Zogning et al (1997), signalent plus de $30 \%$ de carbone organique en dépôt tourbeux dans les sédiments du lac Nyos en plus d'une importante fraction de spicules siliceux et Spongiaires. Ces dépôts tourbeux résultent d'une disparition de la forêt à Podocarpus et Olea capensis vers 2500 ou 2300 B.P. Cette forêt est la source principale du $\mathrm{CO}_{2}$ biologique. En outre, l'on sait qu'à Nyos, le $\mathrm{CO}_{2}$ provient des profondeurs, donc depuis le manteau ( $3^{\text {ème }}$ source du $\mathrm{CO}_{2}$ dissous dans les eaux du lac); et, il est plus efficace que le $\mathrm{CO}_{2}$ biologique. $\mathrm{Ce} \mathrm{CO}_{2}$ remontant des fractures mantelliques doublé de la présence du soufre, de l'anhydride sulfureux a un pouvoir de dissolution plus élevé que le $\mathrm{CO}_{2}$ atmosphérique et biologique. Par, ailleurs , il est admis et connu que les roches riches en carbonate de calcium et de magnésium (péridotites carbonatites dont la composition minéralogique les rend très vulnérables à l'érosion chimique) associés au soufre, aux contraintes tectoniques et aux variations thermiques en montagne sont plus fragiles. Ce qui explique la fragilité du barrage de pyroclastites de Nyos; très riches en ferromagnésiens facilement attaquables par l'eau chargée de $\mathrm{CO}_{2}$.

Dans le cas de Nyos, l'eau de circulation souterraine des grottes, cavernes et fissures contient $2-8 \%$ de $\mathrm{CO}_{2}$ et constitue la première source de dissolution à laquelle s'ajoute le $\mathrm{CO}_{2}$ mantellique plus important. La recharge du $\mathrm{CO}_{2}$ et de l'acide carbonique est fréquente, car celle-ci est la condition sine qua non pour que la saturation soit évitée et que la dissolution puisse continuer.

\section{III-INTERPRETATIONET CONCLUSION}

\section{A) - Impacts de la fracturation et des risques sismiques}

L'intense fracturation relevée peut en cas d'un séisme violent, d'un rejeu ou d'une éruption volcanique provoquer la rupture complète du barrage. Les conséquences de cette rupture seraient incalculables ; notamment, on aurait 6 fois plus de morts que lors de l'éruption de 1986 (qui a fait 1721 morts, 874 victimes hospitalisées et 4434 personnes déplacées) sans oublier d'autres dégâts matériels importants. En effet, dans le cas d'un séisme ou d'une éruption située sur le barrage, les dégâts principaux résulteraient des inondations et des effets directs de l'éruption alors qu'en 1986, les dégâts étaient uniquement liées à l'éruption.. C'est un risque et un grand danger pour les populations en aval et la réserve de Kimbi qui se situe au cœur d'une morphotopographie en alvéoles favorable aux inondations. De plus, les impacts se ressentiront jusqu' au Nigeria situé entre 50 et $70 \mathrm{~km}$ à vol d'oiseau. De plus, l'altitude décroît depuis le lac jusqu'à la frontière avec le Nigeria. En effet, celle-ci passe de 1214-1144m à Nyos \& Acha à $302 \mathrm{~m}$ vers la frontière du Nigeria à Tumbo selon un axe SSE-NNO. Il faut y ajouter le système de fracturation qui crée des couloirs de failles. Ces couloirs précédemment étudiés sont des voies naturelles de canalisation des eaux jusqu'à la frontière avec le Nigeria. On constate, par ailleurs, que l'altitude des reliefs s'abaisse dans ces couloirs. Ensuite, le lac Nyos se situe à la croisée de trois grandes directions de fractures : le système dominé par la $\mathrm{N} 70^{\circ} \mathrm{E}$ hérité du panafricain et guidant les cours d'eau ; mais qui a rejoué et sur lequel s'alignent les lacs Kuk, Befang, Elum, Nyos et Njupi selon une orientation OSO-ENE. Le second système représenté par la faille $\mathrm{N} 148^{\circ} \mathrm{E}$ et sur laquelle s'alignent les lacs Nyos, Oku et Monoun (fig. 1b) correspond à la direction somalienne dominée par des cassures et failles transverses miocènes orientées SENO. La troisième direction d'orientation E-O est constituée de failles listriques récentes pour la plupart et liée au rejeu des vieilles directions panafricaines ainsi qu'à la réactivation des linéaments précambriens. Elles sont franchement $\mathrm{N} 90^{\circ} \mathrm{E}$ (fig. 3). Les autres directions sont secondaires et jouent en distension, notamment, pour les failles verticales $\mathrm{N} 0-5^{\circ} \mathrm{E}$ et $\mathrm{N} 170-175^{\circ} \mathrm{E}$. Or, l'activité tectonique et sismique est permanente sur les Grassfields et sur toute la Ligne Volcanique du Cameroun et elle se double parfois d'éruptions volcaniques : on peut retenir pour mémoire, les secousses telluriques de Magba en 1983 et de Garoua Boulai en 1986 ainsi que les éruptions du Mont Cameroun de mars -avril 1999 et d'avril - mai 2000. MORIN et al. Montrent également que le lac Nyos se situe sur des structures tectoniques actives : un coulissage $\mathrm{N} 70^{\circ}$ E et des décrochements $\mathrm{N} 160^{\circ} \mathrm{E}$.

La fracturation $\mathrm{N} 90^{\circ} \mathrm{E}$ relevée sur la dalle du barrage de pyroclastites se poursuit jusque dans les granites de la bordure $\mathrm{E}$ du lac $\left(\mathrm{N} 30^{\circ}\right.$ et $\mathrm{N} 125^{\circ} \mathrm{E}$ selon Temdjim et Tchoua) où nous avons dénombré 20 grandes fractures en pirogue (photo 4); or dans les pyroclastites qui d'ailleurs s'altèrent très vite, la dynamique de ces fractures est l'écroulement dès lors qu'elles atteignent $20 \mathrm{~cm}$ d'écartement. C'est ce qui explique la rupture de pente de 1,50 $\mathrm{m}$ qui tranche la dalle de pyroclastites de pendage compris entre 10 et $15^{\circ}$ (fig.2c). 


\section{B) - Conséquences et impacts de la dissolution karstique}

La dissolution karstique n'est pas un phénomène simple. Nous venons de montrer qu'elle active à Nyos et que la recharge des eaux en $\mathrm{CO}_{2}$ est permanente à travers le réseau de fracturation qui peut provoquer la rupture du barrage. Pour ce qui est de ses effets sur la rupture du barrage, Corbel (1970) signale que le karst tropical est polymorphe et que sa vitesse d'évolution est de $40-80 \mathrm{~m} /$ millénaire en région humide et 20-40 en région sèche. Contrairement, dans les granitoïdes, l'érosion est de $1 \mathrm{~m}$ tous les $60-80000$ ans (Petit, 1990). Rossi (1980) dans le cas du karst malgache signale une érosion de $135 \mathrm{~m} / 1000 \mathrm{ans}$. Nyos étant une région tropicale humide de montagne (et son barrage de pyroclastites fonctionnant comme un karst), cette dissolution donnerait une vitesse de $0,08 \mathrm{~mm} / \mathrm{an}$ selon les données de Corbel et $0,13 \mathrm{~mm} / \mathrm{an}$ selon celles de Rossi. Or, le barrage de pyroclastites de Nyos date de 500 B.P.; donc la dissolution pourrait être de 40 à $65 \mathrm{~m}$ dans le tout le barrage, non compris le temps nécessaire à l'enclenchement du processus de dissolution. Aka et al. (2001) utilisant la variation de la largeur du barrage signalent une érosion de $0,06 \mathrm{~m} / \mathrm{an}(0,69 \mathrm{~mm} / \mathrm{an})$. Cette érosion et cette vitesse de dissolution qui se tiennent estimées ici ne peuvent être selon ces derniers la cause immédiate d'une rupture du barrage. Cependant, pour nous, elles constituent un processus de préparation du matériel pouvant provoquer des glissements, des éboulements ou des écroulements. En effet, les processus de karstification tendent à accentuer les contrastes entre les roches. La voûte de la grotte ou de la caverne de Nyos peut facilement s'effondrer à cause de la faible résistance des pyroclastites à la pression et de la solubilité élevée liée à la présence des roches riches en carbonates de calcium ou de magnésium (carbonatite, péridotite). Mieux encore, les études d'Alain le Marechal (1976) nous permettent de réaliser que les sources thermominérales camerounaises (notamment celles des régions volcaniques) sont fortement bicarbonatées et magnésiennes et elles sont riches en silice (voire II dans la partie précédente). Les eaux et source du lac Nyos n'échappent pas à ce caractère et la dissolution devient plus forte parce que les eaux circulent au contact du magma basique ou ultrabasique (carbonatite, péridotite). Les effondrements sont consécutifs aux développements des cavités souterraines, à l'abondance des eaux (chargées de $\mathrm{CO}_{2}$ ) d'écoulement souterrain issues du lac ou des infiltrations. Ces effondrements sont susceptibles de provoquer une rupture du barrage qui subit déjà la pression du volume d'eau du lac.

\section{C) - Rôle du volume d'eau}

Si l'eau du sol est riche en $\mathrm{CO}_{2}$ à proportion de $50-200 \mathrm{mg} /$ 1 , à combien plus forte raison l'eau du lac Nyos en contient et ce d'autant plus que le $\mathrm{CO}_{2}$ dissous dans l'eau provient du manteau. Il faut donc compter avec une concentration minimale de $200 \mathrm{mg} / \mathrm{lde} \mathrm{CO}_{2}$, ce qui équivaut à une pression supérieure à $10 / 100^{\mathrm{e}}$. Une rupture dans ce contexte projetterait un minimum de 0,03 à $0,06 \%$ des eaux du lac.

Eno Belinga et Njilah (2001), évaluent de façon modeste la quantité d'eau qui sera déversée en cas de rupture du barrage à 50millions de $\mathrm{m}^{3}$. Cependant, compte tenu de la quantité d'eau du lac (3milliards de $\mathrm{m}^{3}$ ) et de la forte concentration du $\mathrm{CO}_{2}$ mantellique, une simple rupture du barrage concomitamment à d'autres forces et phénomènes conjugués internes au maar ( secousses sismiques, microséismes imperceptibles, upwelling, éruption volcanique ou gazeuse, dissolution karstique préparant le matériel, effondrement de la voûte liée à la dissolution karstique) pourraient provoquer une inondation certes, mais, avec une quantité plus importante d'eau; au minimum $80-100$ millions de $\mathrm{m}^{3}$ qui représenteraient $0,03 \%$ des eaux du lac. Il est admis et connu que les séismes et éruptions au Japon provoquent des raz de marée (tsunamis) catastrophiques; sans dramatiser, ni être catastrophiste, un séisme ou une éruption dans le contexte du barrage de Nyos peut bien provoquer un débordement d'un volume de plus de 200millions de $\mathrm{m}^{3}$ d'eau. En effet, EVANS, (1996) montre qu' une concentration maximale de $0,43 \mathrm{~mol} \mathrm{~kg}-1 \mathrm{de}$ $\mathrm{CO}_{2}$ sous les eaux du lac peut produire un jet d'eau de $150 \mathrm{~m}$ de hauteur. Cette donnée récente d'EVANS confirme ce que MORIN et al. (1986) proposent: à savoir que lors de la catastrophe de 1986, le jet d'eau, liée à l'émission du $\mathrm{CO}_{2}$ a pu atteindre 120-140m au-dessus du lac. L'on sait aussi que l'eau des sources thermominérales arrive en surface par pression hydrostatique et gazeuse.

\section{CONCLUSION}

Les travaux actuels cherchent à réduire le volume d'eau pour minorer les risques de rupture du barrage de pyroclastiques qui apparaissent hachées de failles et qui représentent en dehors du gaz $\mathrm{CO}_{2}$ un second danger potentiel. Dès lors, ils permettent à coup sûr de parer au danger de rupture et de protéger la vie des populations. En effet, l'on se souvient pour mémoire qu'en 1986, 2 semaines avant l'éruption, une équipe d'observateurs de passage avaient entendu les populations se plaindre de phénomènes bizarres qui se passaient dans le lac. D'après celles-ci, les eaux bougeaient d'un côté (communication orale de Dr. Roger NGOUFO). En tenant compte de cette communication, nous préconisons si possible l'utilisation 
de la mémoire collective des rescapés pour maîtriser et comprendre davantage le mécanisme du lac Nyos. Depuis quelques années, il existe un mouvement de retour de la plupart des populations installées à Kimbi. Ce mouvement trouve son origine et son explication dans les liens traditionnels inoubliables des populations sinistrées avec la terre de leurs ancêtres, seul héritage et seul moyen d'identité culturelle et d'épanouissement. Il faut cependant ajouter à cela la négligence des autorités administratives et politiques envers les rescapés abandonnés à leur sort et qui préfèrent retourner mourir. Par conséquent, il est fort possible d'utiliser la mémoire collective de ceux-ci comme moyen parmi tant d'autres à conjuguer pour enrayer à l'avenir de telles catastrophes. Le manque d'infrastructures routières et de ponts constitue un autre danger puisque l'accès à Nyos n'est pas facile en saison pluvieuse et constituerait un risque potentiel en cas de cataclysme naturel parce que les équipes de secours d'urgence ne pourront avoir accès au site du sinistre. Ainsi, on ne peut évaluer ces risques d'inondation sans tenir compte de tous ces paramètres: les populations et leur patrimoine ainsi que de la faune sauvage. Il s'agit là de quelques mesures qui pourraient être mises en place pour réduire ou juguler les risques de catastrophes au Lac Nyos.

\section{BIBLIOGRAPHIE}

AKA F.T., KUSAKABEM. \& NAGAO K, (2001). - New KAr ages for Lake Nyos maar, Cameroon. Implications on hazard evaluation. In Géosciences du Cameroun; vol.1, $\mathbf{N}^{\circ} \mathbf{1 A}$, pp. 25-26

BELINGA S.M. \& NJILAH I. K., (2001). - From Mount Cameroon to Lake Nyos. Les Classiques Camerounais, Yaoundé, 128p.

CHEVRIER R.M., (1990). - Lake Nyos: phenomenology of the explosive event of 30 December 1986. Journal of Volcanology and Geothermal Research, 42, 387-390.

CORBEL J., (1957). - Les karsts du Nord-Ouest de l'Europe et de quelques régions de comparaisons. Lyon, Institut d'études Rhodaniennes.

CORBEL J., (1957). - « Karsts Haut-Alpins ». Revue de Géographie de Lyon; XXXII, N² 2, pp. 135-158.

CORBEL J., (1962). - Neiges et Glaciers. Armand Colin, $224 \mathrm{p}$.

CORBEl J. \& MUXART R., (1970). - Karsts des zones tropicales humides. Zeitschrift Für Geomorphologie; B14, H4, pp. 411-474.

DERRUAU M., (1974). - Précis de Géomorphologie. Masson, Paris, 453p.

EQUIPE DE PROJET « Orgues de Nyos », (1992). - Rapport sur l'étude de faisabilité du programme «Orgues de Nyos » menée sur site en mars-avril 1992. Résultats, 19p.
EVANS W.C., (1996). - Lake Nyos: knowledge of the fount and the cause of disaster. Nature, 379, 21-22.

FOUCAULT A. \& RAOULT J.F., (1995). - Dictionnaire de

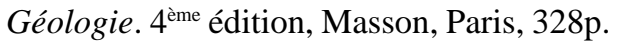

FREETH S. J., (1988). - When the lake Nyos dam fails, there will be serious flooding in Cameroon and Nigeria - but, when will it fail? Eos, Transactions American Geophysical Union; 69, 776-777.

FREETH S. J., (1990). - The anecdotal evidence, did it help or hinder investigation of the lake Nyos gas disaster. Journal of Volc. and Geoth. Res.; 42, 373-380.

FREETH S. J.,(1992). - The lake Nyos gas disaster. In: Natural hazards in West and Central Africa (edited by FREETH S.J.) pp. 63-82. Vieweg, Braunschweig.

KUSAKABE M.,OSHUMI T. \& ARAMAKI S., (1989). The lake Nyos gas disaster: chemical and isotopic evidence in water and dissolved gases from three Cameroonian crater lakes; Nyos, Monoun and Wum. Journal of Volc. and Geoth. Res.; 39, pp. 167-185.

KUSAKABE M., TANYILEKE G.Z., McCORD S.A. \& SCHLADOW S.G., (2000). - Recent pH and $\mathrm{CO}_{2}$ profiles at Lakes Nyos and Monoun Cameroon: implications for the degassing strategy and it numerical simulation. Journal of Volc. and Geoth. Res., 97, pp. 241-260.

LEENHARDT O., (1991). - La catastrophe du lac Nyos. Aspects scientifiques. Géologues $\mathrm{N}^{\circ}$ 96, Paris, pp. 13-24.

LOCKWOOD J. P. \& RUBIN M., (1989). - Origin and age of the lake Nyos maar, Cameroon. Journal of Volc. And Geoth. Res.; 39, 117-124.

McKIE R., (2000). - The World most deadly bubble an explosion of lethal gas from a Cameroon lake killed 1700 people. Scientists are racing to prevent a worse disaster. Reports Robin McKIE, august 20, 2000 in Yahoo mail, 3p.

MORIN S. \& PAHAI J., (1986). - La catastrophe du lac Nyos (Cameroun). Revue de Géographie du ameroun. vol. VI, $\mathrm{N}^{\circ}$ 2, pp.81-105.

NJILAH I.K., (1991). - Geochemistry and petrogenesis of Tertiary-Quaternary volcanic Rocks from Oku-Ndu area, NW Cameroon. Unpublished Ph. D Thesis, University of Leeds, 345p.

PETIT M., (1990). - Géographie physique tropicale. Approches aux études physiques du milieu. Morphogenèse-Paysages. Karthala-ACCT, paris, 351p.

QUINIF Y., (1985). - Une morphologie karstique typique en zone intertropicale: les Karsts du Bas-Zaïre. Karstologia, 6, pp. 43-52.

ROSSI G., (1980). - L'extrême Nord de Madagascar. Edisup, 435p. 
SAMO Y.,KUSAKABE M., HIRABAYASHI J., NOJIRI Y., SHINOHARA H., NJINET. \& TANYILEKEG.Z.,(1990)

- Helium and carbon fluxes in lake Nyos, Cameroon: constraints on next gas burst. Earth and Planetary Science Letter. 99, pp. 303-314.

SHANKLIN E., (1989). - Exploding lakes maleficent water in Grassfields legends and myth. Journal of Volc. and Geoth. Res. 39, pp. 233-234.

SIGURDSSON H., DEVINE J.D., TCHOUA F.M., PRESSER T.S.,PRINGLEM.K.W. \& EVANS W.C., (1987). - Origin of the lethal gas burst from lake Monoun Cameroon. Journal of Volc. and Geoth. Res. 31, pp.1-16.

TAZIEFF H., FAIVRE-PIERRET R.X. \& LE GUERN F., (1986). - La catastrophe de Nyos, République du Cameroun. Ministère de la Coopération, $75 \mathrm{p}$.

TAZIEFF H., (1989). - Mechanism of the Nyos carbon dioxide disaster of so-called phreatic steam eruption. Journal of Volc. and Geoth. Res. 39, pp.109-116.

TEMDJIM R. et TCHOUA F.M. (1991). - Cadre géologique de la région du lac Nyos. Revue de Géographie du Cameroun. vol. $\mathbf{X} \mathrm{N}^{\circ} 1$, pp. 32-38.

TRICART J., (1977). - Géomorphologie Dynamique Générale; Paris, SEDES, 345p.

WANDJI P., (1985). - Contribution à l'étude pétrologique et géochimique des projections volcaniques de la région de Foumbot. Université de Yaoundé, Thèse de $3^{\text {ème }}$ cycle, $159 \mathrm{p}$.

WILLIAMS P. W., (1972). - Morphometric analysis of polygonal karst in New Guinea. Geological Society of America Bulletin; vol. 83, pp. 761-796.

ZOGNING A., GIRESSE P., MALEY J. \& GADEL F., (1997).

- The late Holocene palaeoenvironment in the lake Njupi area, West Cameroon: implications regarding the history of Lake Nyos. Journal of African Earth Sciences, vol. 24, $\mathrm{N}^{\circ}$ 3, pp. 285-300. 